\title{
APPLICATION OF 3D-PMD VIDEO CAMERAS FOR TASKS IN THE AUTONOMOUS MOBILE ROBOTICS
}

\author{
Alexander Prusak ${ }^{1}$, Hubert Roth ${ }^{2}$, Rudolf Schwarte ${ }^{3}$ \\ ${ }^{12}$ Institute of Automatic Control Engineering, University of Siegen, Germany \\ ${ }^{3}$ Institute of Information Processing, University of Siegen, Germany \\ ${ }^{1}$ alexander@prusak.de, ${ }^{2}$ hubert.roth@uni-siegen.de, ${ }^{3}$ rudolf.schwarte@uni-siegen.de
}

\begin{abstract}
Fast Localisation is a basic function within the mobile robotics. Beside the odometrie, mainly image sensors are used. This paper shows a short overview of the image sensors used for it. Outgoing from the 2D, 3D laser scanner, over 2D mono camera and 2D stereo camera, up to the omni-directional 2D camera are described problems, which appear in recording and modelling of the robot environment with such sensors. In this paper in comparison to the classical sensors a completely new 3D-PMD video camera is introduced as a more efficiently image sensor. This sensor can not only record the robot environment as normal 2D image but delivers furthermore additionally with the depth information into a three-dimensional video image. Concerning of speed and accuracy, new possibilities are opened in object and obstacle recognition, localization and autonomous map generation, within the mobile robotics. Copyright (C) 2005 IFAC
\end{abstract}

Keywords: Autonomous mobile robots, Robot navigation, Robot vision, 3D-PMD video camera, Image sensors, Image processing, Image analysis, map building, SLAM.

\section{INTRUDUCTION}

The nature has developed many ways for successful $3 \mathrm{D}$ navigation from the insects up to the mammals. The research in this technology for use in mobile robotics suggests an enormous development in mobile robots in the next time. Mobile robots are to be used for exploration, search, rescue, service, etc. and need a certain degree of autonomy. At present, the autonomy is solved unsatisfactorily within mobile robots. The crucial step is the fast acquisition of the relevant 3D data from the 3D environment. Part 2 of this paper represents the typically used sensors. Part 3 shows a completely new method for recording this $3 \mathrm{D}$ data by using the so-called $3 \mathrm{D}$ PMD video camera.

\subsection{Types of mobile robots.}

Present the main operational area of mobile robots lies in the industry as so-called „Autonomous Guided Vehicle" (AGV, see Fig. 1). AGVs used in factory buildings, for transport of materials and work pieces in the production area without a driving operator.
Today, also in private sectors, so-called service robots (Fig. 1) are in the employment, e.g. vacuum cleaners (Kärcher), lawnmowers (Husqvarna), sweeping machines (Hefter Cleantech), safety and guard robot (Robowatch), museum robots, OPEL robot and Care-O-Bot (Fraunhofer IPA) (Fig. 2), etc. The most popular and technologically ambitious mobile robots are the "pathfinder", mars exploration robots „Spirit“ and „Opportunity“ (NASA).

\subsection{Working area of mobile robots.}

Most of the above-described mobile robots exclusively work on even surfaces (2D environment) within buildings (indoors). Only a few applications dare themselves into the free hilly area (3D environment, outdoor). At present, still no useful robot exist, which can navigate e.g. within a building, over several floors, connected with stairs and elevators (indoor, 3D environment) (Fig. 3). The reason is, that beside the mobile robot's construction, the localisation and navigation in its surrounding field has eminent importance 

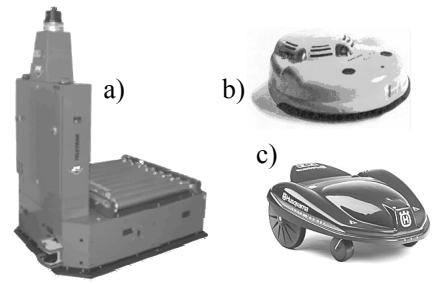

d)

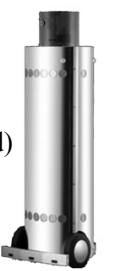

Fig. 1. Mobile Robots: a) Autonomous Guided Vehicle, AGV, (Eilers-Kirf), b) Vacuum cleaner (Kärcher), c) Lawnmower (Husqvarna), d) Safety and guard robot (Robowatch).
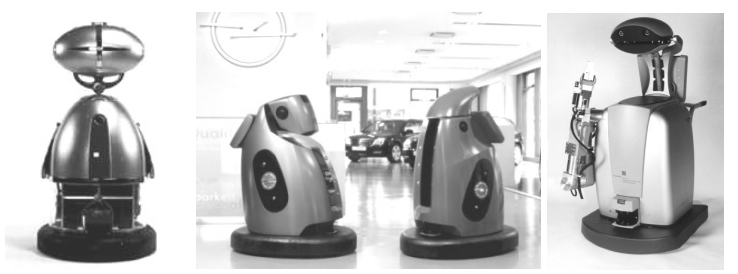

Fig. 2. Museum robot (1.), OPEL robot (m.) and Care-O-Bot (r.) (Fraunhofer IPA).
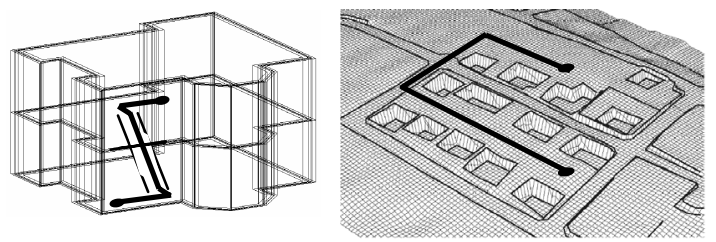

Fig. 3. Robot drives in a 3D environment (indoor and outdoor area).

\subsection{Basic functions, navigation and services.}

First, a mobile robot must own certain basic functions for measurement and control, in order to do any kind of services. The object recognition, obstacle recognition and obstacle avoidance belongs to these basic functions. Thus the simple service ,search" can be already realized. The robot looks for a certain object and remains standing at its location. As the further basic function the localization is mentioned. Localization means to know always the position and orientation of the mobile robot, including its inclination within the $3 \mathrm{D}$ environment.

Based on the basic functions described above the service „exploration” can be implemented. Exploration means, to move in an unknown area and also to explore this environment. During exploring, significant objects and characteristics are registered into a virtual map as natural landmarks of the unknown area. Localization with simultaneous map generation is called SLAM (Simultaneous Localization and Map Building).

For exploration tasks, the robot can carry out purposeful or coincidental movements. During a purposeful robot movement, the mobile robot drives on a given path in the unknown area, which is in particular suitable for the outdoor area. For the indoor area the coincidental movement is suitable. Here, the starting point of the mobile robot is defined. The further robot movement is rather coincidentally by changes of direction at obstacles and through driving along walls.

A defined path, a mobile robot has to drive, is given by the starting point, several intermediate points and the end point. The mobile robot must successively drive to these points. With the path description and the localization a suitable control algorithm navigates the mobile robot, if it is on the correct points according to the path. According to this, the control algorithm corrects the position of mobile robot through changes its speed and direction. With the appearance of an obstacle, discovered by the object and obstacle recognition sensors, the navigation of the mobile robot must avoid collisions. It must be examined if the obstacle performs a movement itself. In this case the mobile robot can interrupt its travel until the obstacle leaves the path. Otherwise, the mobile robot would leave its given path with the obstacle avoidance function, drives around the obstacle, finally drives back to the original path again and continues the travel. With the availability of a map, a priori given or autonomously generated, one or more paths can be drawn into this map. With all above-described implementations, now useful services can be created, like courier service, inspection, observation, cleaning, etc.

\section{LOCALISATION}

The localization determines position and orientation including the inclination of a mobile robot according to the starting point. In this so-called relative localization it is enough to know the changes of position and orientation from the start of the travel. A service robot, e.g. to clean always the same area, needs only the changes in relation to the start point. It behaves differently, if position and orientation data are used for other systems without common point of reference. In this case are absolute values necessarily, like lengthening and degrees of latitude. For example, of a mine search robot the positions of found mines must be indicated in absolute values, for the production of target-plans.

For the determination of position and orientation of the mobile robot, at present numerous not very accurate sensors are used, like wheel encoder, magnetic compass, gyroscope compass, inclination sensor, acceleration sensor, LPS (local positioning system) or GPS (global positioning system). The data of these sensors can be fused e.g. on the basis of Kalman filter algorithms to receive their optimal developing of values (VDI/VDE, 2003; Girard, et al., 2001; Ashokaraj, et al., 2002; Bing-Yung Chee, et al., 1996; Rusu, et al., 2002). 


\subsection{Localization with odometrie.}

A simple and often realized method is the odometrie, where the evaluation of the wheel encoder impulses is counted for localization (Sang-Gyum, et al., 2001). From the geometry of the wheels, their distance to each other and the measured wheel encoder impulses sequentially the new position and orientation of the mobile robot is computed. Localization with such wheel encoders represents an integral method, in that the incremental changes are sequentially added for the location and also all errors from the starting point. Errors, caused e.g. by chutes and sliding of the drive wheels or driving over stage, are considered in the computation and falsify the location data of the mobile robot.

\subsection{Localization with $2 D$ and $3 D$ laser scanner.}

2D laser scanners are contact less working measuring systems, which scan in two dimensions their environment with a laser beam. A rotary mirror diverts the laser beam, which is reflected at the object surface in the environment. Over a running time measurement can be determined the distance to the object surface points. Together with the current rotation position of the mirror the points of impact in polar coordinates can be represented. Laser scanners are available from different companies like e.g. „Sick” (Sick). For example the laser scanner LMS200 of the company „Sick” gropes a range from $180^{\circ}$ with an incrementation of $0.5^{\circ}$. The distance accuracy is to $15 \mathrm{~mm}$ and the range with $+/-8$ meters.

With such laser scanners, mounted on mobile robots, its location can be determined. So-called artificial landmarks (e.g. reflectors) are measured, which are fixed along the way and whose positions are known. At least three of such defined objects must be detected and with the help of the triangulation procedure the position and the orientation of the mobile robot can be computed (Nehmzow, 2002). In opposite to the odometrie, this represents a kind of localization by a non integrative procedure. By direct measurements within the real environment, the socalled absolute localization can be realized.

Instead of the artificial landmarks also natural landmarks can be used, e.g. corners, edges and also geometrical forms. This procedure is particularly suitable in localization with the help of a map, in which such characteristics are stored, e.g. CAD map with sketch of a building.

In principle a laser scanner acquires the environment of a mobile robot only in an individual level, i.e. it presents thereby only 2D depth data. By additional mechanical swivelling and pitch motions of the entire laser scanner system, several levels can be acquired, so that the robot environments are present now in 3D depth data. Due to the mechanical deflection movements, the collection of an individual
$3 \mathrm{D}$ image, with a scan angle range of $150^{\circ} \times 90^{\circ}$ needs between 4 and 12 seconds depending upon resolution (Surmann, 2001). For the on-line collection of the 3D environment data and the localization in real time, this system is too slow.

\subsection{Localization with $2 D$ video camera.}

In opposite to the 3D laser scanner (max. 1/4 images per second) a video camera acquires the environment of a mobile robot with a frame rate of approximately 25 images per second. In the simplest case it is a normal mono camera this can provide only 2D images. With this camera the depth data of the $3 \mathrm{D}$ robot environment cannot be acquired, but these are important for the localization. For the reconstruction of the depth data from 2D image sequence, the stereo procedure in two different ways can be used.

First, two mono cameras fixed as a stereo camera system, are mounted on a mobile robot. Both cameras are fixed in a defined distance side by side and parallel to their optical axis. Now by the stereo double images the depths information can be calculated for each pixel. Within the stereo double image, it is searching for corresponding 2D image elements, e.g. on a sufficiently rough texture, for calculating the distance with e.g. the procedure of the surface-based cross correlation for each pixel.

But this stereo procedure can also be used with a single mono camera for getting the depth information. The mono camera supplies sequential images, they are recorded on different positions during a robot movement. From each sequential image pair the depth information can be evaluated with the above described stereo procedure. With the stereo camera system, the two cameras have a defined position side by side, whereas the mono camera has individual camera positions, which are different and dependent on direction and speed of the mobile robot movement.

\section{THE 3D-PMD VIDEO CAMERA}

The University of Siegen and Center for Sensor Systems (ZESS) develops a new so-called 3D-PMD video camera (Photonic Mixer Device) (Fig. 4).

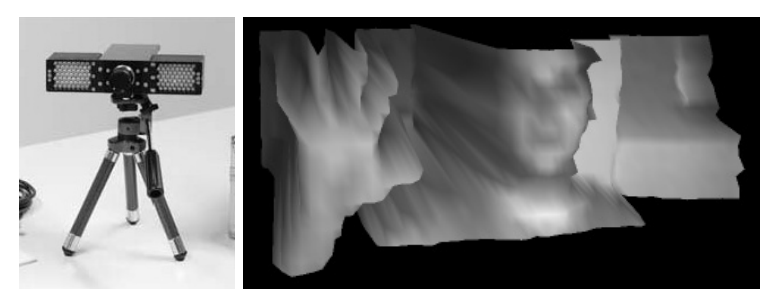

Fig. 4. 3D-PMD video camera with $64 \times 16$ pixel (1), delivers $3 \mathrm{D} / 2 \mathrm{D}$ video pictures, encodes in depths and grey value data $(r)$. 
This camera can acquire a normal 2D image data and in addition delivers pixelwise directly the depth or distance information of a 3D scene (Schwarte, et al., 2003). It consists of an external transmission unit (LED array) and an internal chip of PMD pixels. Each individual PMD pixel acts similar to a laser radar receiver, with the Time-of-Flight (TOF) principle, whereby the running time is measured of the modulated light signal from the transmitter (LED array) to the reflecting object and back to the receiver. The distance information lies in phase of the reflected modulated light signal.

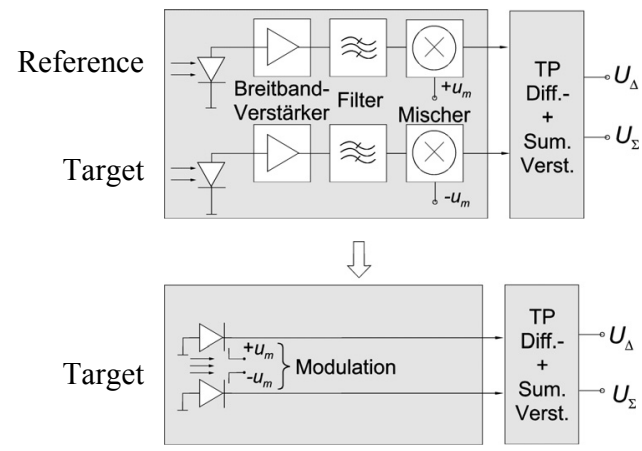

Fig. 5. Conventional receiver (above) and PMD receiver (below).

The function is illustrated at a simple conventional receiver (Fig. 5, above). This receiver exhibits a relatively high time drift and needs for their compensation an almost identical reference receiver. Every channel consists of a photodiode, ambitious HF (high frequency) amplifiers, filters and a mixer. The photodiode converts the received optical signal into an appropriate light current. The light current is amplified and arrives at the electrical-electrical mixer. Here a comparison of the amplitudes and phases with the transmitter modulation signal acts. This determined phase change between the sent and received signal, represents the echo delay time and shows the depth of the pixel in the appropriate area.

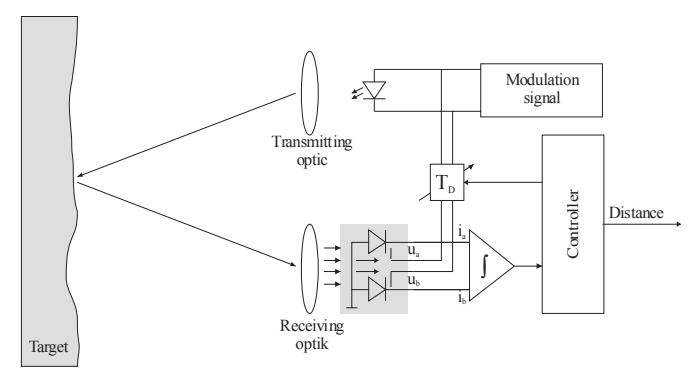

Fig. 6. Time-of-Flight (TOF) measurement with one PMD pixel.

In relation to a conventional receiver, in the PMD receiver (Fig. 5, down) the reference channel can be omitted. Only the new element PMD remains remaining, practically an inherently mixing photodiode with common photo sensitive area and with two outputs (two selection cathodes) exists. The modulated light current of the delayed echo signal is conducted over the voltages $+u_{m}$ and $-u_{m}$ to the steering photo gates (Fig. 5, below; Fig. 6), alternatively on or other output gate and demodulated it in such a way. The PMD technology eliminates all HF components described above, amplifier, mixers etc. and their error influences. The difference of the push-pull channels results in the phase and thus the 3D information. The summation of this results in the 2D intensity information (greyscale). Besides the reducing of the expensive HF components and their drift, noise and dynamic problems, the receiver shrinks on image pixel size and permits economical production of these so-called Photo Gate PMDs (PG$\mathrm{PMD}$ ) in standard CMOS technology ( $\mathrm{Xu}$, et al., 1999).

\section{$3.13 D-2 D$ mixed camera.}

Such a 3D-PMD video camera delivers 3D depth images and beyond also 2D greyscale images of the recorded scene. It is often an advantage to get $2 \mathrm{D}$ colour images instead of the 2D greyscale images, whereby a data format is available in particular for robotics: a PMD image, in which each pixel contains beside the colour values (red, green, blue) additionally the depth information. In the form of a so-called 3D-2D mixed camera, different possibilities are presented here, to get 3D depth images and pixelappropriate 2D colour images of a recorded scene.

3D-PMD video camera with $2 \mathrm{D}$ video camera: The simplest method consists of fixing a 3D-PMD video camera and a 2D video camera parallel and in the defined distance to their optical axis (Fig. 7, left). The pixel-appropriate allocation of the respective image data is processed by software. Here are the different camera parameters considered, like focus, lens, flare angle, etc.

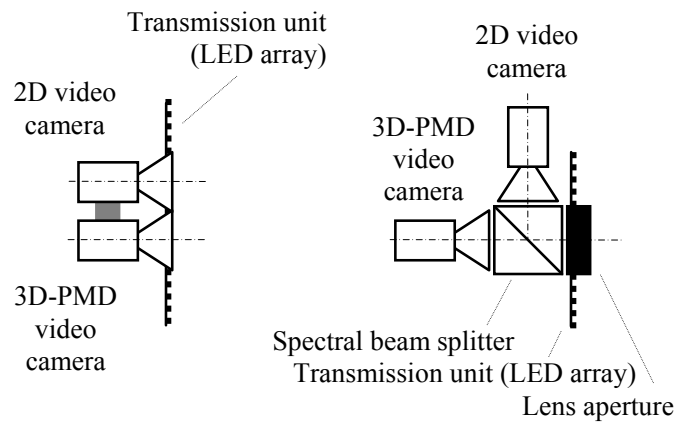

Fig. 7. 3D-2D mixed camera without (left) und with (right) spectral beam splitter.

3D-PMD video camera with $2 \mathrm{D}$ video camera and spectral beam splitter: Such a camera consists of a 3D-PMD video camera, a 2D video camera and a spectral beam splitter (Fig. 7, right). The modulated LED light falls through the lens aperture, together with the environmental light of the scene. It is divided at the spectral beam splitter (Edmund; Sill) and arrives in the appropriate camera depending on the wavelength. The recorded images and their pixelappropriate allocation are respectively determined by the mechanical structure of this camera. 
3D-2D mixed camera with PMD and CCD pixel chip: Such a camera could be developed similarly like the 3D-PMD video camera without an additional 2D camera. However the optical chip would consist of pixel, which contains a PMD and a CCD segment. The selection electronics on this chip could spend per pixel 4 data, like 3D depth information, red, green and blue value.

\subsection{Localization with 3D-PMD video camera.}

Localization of a mobile robot with the 3D-PMD video camera can also be realized with an a priori given or autonomously generated map. A relation between the global 2D map and the 3D snapshot of the 3D-PMD video camera must be found. At first the 3D snapshot must be converted into a $2 \mathrm{D}$ snapshot, whereby all heights data of the natural landmarks are projected on a floor space. The 3D depth data are further available. These projected natural landmarks, like corners, edges and possibly connected by lines, represent a $2 \mathrm{D}$ sub map. The localization process works now in such a way that a suitable image processing system searches for this 2D sub map with their characteristics and objects inside the global 2D map. The adaptation of the 2D sub map into the global 2D map is done by translation, rotation and possibly scaling, also socalled image matching. Translation and rotation are the searched values for position and orientation of the mobile robot.

\subsection{SLAM with 3D-PMD video camera.}

On the basis of natural landmarks, the mobile robot generates a virtual map of its environment automatically. Natural landmarks differ in their characteristics, like geometry, texture, colour and position (Jähne, 2002). A direct and fast production of the distance information is crucial for the SLAM procedure. For the generation of a map of the robot environment the 3D-PMD video camera can be used very well. Thereby a 3D-PMD video camera (or 3D2D Mixed camera) showing in the main direction of motion is mounted on the mobile robot. Maps are generated by the robots movement and with simultaneously recorded 3D snapshots from different positions of the environment. The mobile robot begins at a defined starting point and drives into the still unknown area. During the exploration of the unknown area the movement of the mobile robot is adapted so that sequential images of the 3D-PMD video camera provide a sufficient overlap. By the 3D depth data of the 3D-PMD video camera and this overlap of images the new position and orientation of the mobile robot can be calculated. The calculated new position and orientation of the mobile robot and the data-reduced 3D snapshot of the environment gets together registered into the virtual map with consideration of the overlap. By this technique, the entire virtual map grows piecewise. On the basis of the 3D snapshots of the 3D-PMD video camera, now $3 \mathrm{D}$ maps can be generated.

\subsection{SLAM with omni-directional 3D camera.}

The mobile robot uses here a camera system looking in all directions, which consists of a 3D-PMD video camera (or 3D-2D Mixed camera) and a sealed cone (Fig. 8).

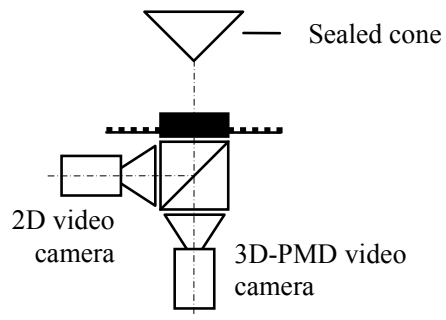

Fig. 8. Omni-directional 3D camera.

An omni-directional 3D camera can be used for the localization, generation of map and navigation. Such a camera can record spatially the entire environment of the mobile robot as $360^{\circ}$ panorama images. This panorama image is available as $2 \mathrm{D}$ and as $3 \mathrm{D}$ image and must be equalized computationally according to the contour of the sealed cone. During the map generation, several of such panorama images must be recorded from different locations and can merge the data to $3 \mathrm{D}$ maps. Panorama image recordings from different locations of the environment avoid covered and unidentified areas. Thus a location-independent virtual 3D map can be generated.

\section{CONCLUSION}

The 3D-PMD video camera have at present the fellow characteristic: Resolution: $64(\mathrm{~h}) \times 16(\mathrm{v})$ PMD pixel, Z-Resolution: $>6 \mathrm{~mm}$, Field of View: $34^{\circ}(\mathrm{h}) \times 12^{\circ}(\mathrm{v})$ full angle, Frame Rate: up to $50 \mathrm{fps}$, Data Format: 8 bit for 3D and 8-bit for greyscale, Digital Interface: Fire Wire (IEEE 1394a) and Ethernet (IEEE 802.3u) (PMDTechnologies). In the near time the Frame Rate have 100fps and the PMD pixel array will increase up to $120 \times 160$ and VGA format later. This 3D-PMD video camera records a robot environment as $3 \mathrm{D}$ depth image and $2 \mathrm{D}$ greyscale image. Concerning of speed and accuracy, new possibilities are opened in object and obstacle recognition, localization and autonomous map generation, within the mobile robotics. With this camera it is possible to produce also 3D maps. A fast localization and exact maps are the basis for real services inside the $3 \mathrm{D}$ environment, like exploration, search, rescue, observation, inspection, cleaning, etc., those could be the future task for mobile robots.

\section{REFERENCES}

Ashokaraj, I.; Silson, P.; Tsourdos, A. (2002): Application of an extended Kalman filter to multiple low cost navigation sensors in wheeled 
mobile robots. Proceedings of IEEE Sensors 2002.

Bing-Yung Chee; Lang, S.Y.T.; Tse, P.W.T. (1996): Fuzzy mobile robot navigation and sensor integration. Fuzzy Systems, 1996. In: Proceedings of the Fifth IEEE International Conference on, Volume: 1, 8-11 Sept. 1996. Page(s): 7 -12 vol. 1.

Edmund Industrie Optik GmbH, Spectral beam splitter, www.edmundoptics.de

Eilers \& Kirf GmbH, Autonomous Guided Vehicle (AGV), www.eilers-kirf.de

Fraunhofer IPA, Museum-Robot, Opel-Robot and Care-O-Bot, Fraunhofer Institute IPA in Stuttgart, www.ipa.fhg.de

Girard, A.R.; de Sousa, J.B.; Hedrick, J.K. (2001): An overview of emerging results in networked multi-vehicle systems. Decision and Control, 2001. In: Proceedings of the 40th IEEE Conference on, Volume: 2, 4-7 Dec. 2001, Page(s): 1485 -1490 vol.2

Hefter Cleantech GmbH, Sweeping machines, www.hefter.de/cleantech

Husqvarna Inc., lawnmowers, www.husqvarna.com

Jähne, Bernd (2002): Digitale Bildverarbeitung. Springer ISBN: 3-540-41260-3, XII, 618 S., 248 Abb., 1 CD - ROM, 5. überarbeitete und erweiterte Aufl., 2002.

Kärcher GmbH, Vacuum cleaners, www.kaercher.de

NASA, Mars Exploration Rover Mission, marsrovers.jpl.nasa.gov

Nehmzow, Ulrich (2002): Mobile Robotik - Eine praktische Einführung. Springer, ISBN: 3-54042858-5, XVI, 264 S., 137 Abb., 2002.

PMDTechnologies GmbH, 57076 Siegen, Germany, 3D-PMD video camera, www.pmdtec.com

Robowatch GmbH, Safety and guard robots, www.robowatch.de

Rusu, P.; Petriu, E.M.; Whalen, T.E.; Cornell, A.; Spoelder, H.J.W. (2002): Behavior-based neurofuzzy controller for mobile robot navigation. Instrumentation and Measurement Technology Conference, 2002. IMTC/2002. In: Proceedings of the 19th IEEE, Volume: 2, 21-23 May 2002. Page(s): 1617 -1622 vol. 2.

Sang-Gyum, Kim; Hee-Chang, Moon; Chang-Man, Kim; Young-Hoon, Park; Jung-Ha, Kim (2001): Sensor guided unmanned vehicle system for the tele-operation. CA Conference paper (C) / IASTED. - In: Proceedings of the IASTED International Conference. Robotics and Applications / Hamza, M.H. - Anaheim, CA, USA, USA: ACTA Press, 2001, ii+239 p.32-8

Schwarte, R.; Buxbaum, B.; Ringbeck, Th.; Xu, Z.; Gollewski, T. (2003): 3-D-Kamera für Mobile Telerobotik und Telemanipulation. In: Telematik 2003, VDI-Berichte 1785, Fachtagung, Juni 2003, Siegen, ISBN 3-18-091785-7, S. 105 114.

Sick AG, Laser scanner, www.sick.de

Sill Optics GmbH \& Co. KG, spectral beam splitter, Wendelstein, www.silloptics.de

Surmann, Hartmut; Lingemann, Kai; Nüchter, Andreas; Hertzberg, Joachim (2001): Aufbau eines 3D-Laserscanners für autonome mobile Roboter. GMD Report 126, GMD Forschungszentrum Informationstechnik $\mathrm{GmbH}$, 2001.

VDI/VDE (2003), Gesellschaft Mess- und Automatisierungstechnik: Telematik 2003. Tagung Siegen, 17. und 18. Juni 2003 / VDI/VDEGesellschaft Mess- und Automatisierungstechnik. - VDI-Verl., Düsseldorf, 2003. - 235 S.: Ill., graph. Darst. - ISBN: 3-18091785-7 A. - (VDI-Berichte; 1785)

$\mathrm{Xu}, \mathrm{Z}$. (1999): Investigation of 3D-Imaging Systems based on Modulated Light and Optical RFInterferometrie (ORFI). Dissertation, INV, Universität Siegen, 1999 